\title{
TOPOLOGIES OF GROUP ALGEBRAS AND A THEOREM OF LITTLEWOOD
}

\author{
BY \\ SIGURĐUR HELGASON
}

1. Introduction. An important problem in Fourier analysis is that of investigating the relationship between the "size" of a function and the "size" of its Fourier transform. The present paper can be regarded as a contribution to this problem.

The classical Fourier transformation establishes a one-to-one correspondence between the set $L^{1}(R)$ of all Lebesgue integrable functions $\left.{ }^{1}\right)$ on the real line $R$ and certain set of bounded continuous functions on $R$. This correspondence gives rise to two different metrics on the set $L^{1}(R)$, namely one given by assigning to each $f \in L^{1}(R)$ the usual $L^{1}$-norm and the other by assigning to $f$ the maximum of its Fourier transform $F(u)=(2 \pi)^{-1 / 2} \int f(x) e^{-i x u} d x$. This last metric coincides (as follows easily from the Plancherel formula) with the metric obtained by the norm of the convolution operator $g \rightarrow f * g$ on the Hilbert space $L^{2}(R)$.

Even when $G$ is an arbitrary locally compact unimodular group we can in this way assign two natural topologies to the set $L^{1}(G)$ of Haar integrable functions. The first is given by the $L^{1}$-norm $f \rightarrow \int|f(x)| d x$ ( $d x$ denoting left invariant Haar measure) and the other by the spectral norm $f \rightarrow\left\|L_{f}\right\|$ where $L_{f}$ denotes the convolution operator $g \rightarrow f * g$ on $L^{2}(G)$. A linear operator on $L^{1}(G)$ we shall call spectrally continuous if it is continuous from the spectral norm topology to the topology of the $L^{1}$-norm.

The purpose of the present paper is to determine the spectrally continuous operators that commute with all right translations on $G$. The answer breaks up into two entirely different cases, namely the compact case and the noncompact case.

I. $G$ is compact. Here the operators in question are precisely left convolutions by $L^{2}$-functions. (Theorem A). The proof is obtained by a study of certain lacunary Fourier series on the product group $G=\prod_{x} U\left(d_{x}\right)$. Here $\chi$ runs over all equivalence classes of irreducible unitary representations of $G$ and $d_{\chi}$ denotes the degree of $\chi$. In a later publication we intend to discuss more fully lacunary Fourier series on noncommutative groups. In the classical case when $G$ is the circle group our result yields a slight sharpening of the following well known theorem of Littlewood: If $\sum a_{n} e^{i n x}$ is a Fourier series which remains an $L^{1}$-series after arbitrary change of the arguments of the Fourier coefficients $\left(a_{n}\right)$ then $\sum\left|a_{n}\right|^{2}<\infty$. The case of an abelian group was treated

Received by the editors April 30, 1956.

(1) As usual, functions which differ only on a set of measure 0 are identified. 
by different methods by A. Grothendieck [5] and by the author [6]. I am much indebted to Dr. Grothendieck for interesting discussions about the noncommutative case.

II. $G$ is noncompact. Provided $G$ satisfies certain minor restrictions the only spectrally continuous operator commuting with right translations is 0 (Theorem B). The proof is obtained by using the structure theory of connected locally compact groups to construct certain relative identities in the group algebra $L^{1}(G)$ and then use the generalized Plancherel theorem to estimate the spectral norm. Since the Plancherel theorem is based on von Neumann's reduction theory we assume in this part that $G$ is separable.

If $G$ is abelian the other restrictions on $G$ can be relaxed somewhat as shown in [6], where spectrally continuous operators are studied for commutative Banach algebras.

2. Notations and definitions. Throughout this paper $G$ denotes a locally compact unimodular group, and $L^{p}(G)$ for $1 \leqq p \leqq \infty$ the Banach space of $p$ th power integrable complex valued functions on $G$, equipped with the norm $\|f\|_{p}=\left(\int|f(x)|^{p} d x\right)^{1 / p},\|f\|_{\infty}=$ ess. sup. $|f(x)|$. Here $d x$ denotes the Haar measure on $G$ which will be assumed normalized when $G$ is compact. The term measure will be used in the sense of Bourbaki [2].

If $\mu$ is a bounded measure on $G$ we write $L_{\mu}$ and $R_{\mu}$ for the bounded $\left({ }^{2}\right)$ operators on $L^{p}(G)$ defined by $L_{\mu} f=\mu * f$ and $R_{\mu} f=f * \mu$ where $(\mu * f)(x)$ $=\int f\left(a^{-1} x\right) d \mu(a)$ and $(f * \mu)(x)=\int f\left(x a^{-1}\right) d \mu(a)$. If $\mu$ is a point measure $g \rightarrow g(a)$ we write $L_{a}$ and $R_{a^{-1}}$ instead of $L_{\mu}$ and $R_{\mu}$, and if $\mu$ is absolutely continuous with respect to Haar measure and has derivative $f \in L^{1}(G)$ we write $L_{f}$ and $R_{f}$ instead of $L_{\mu}$ and $R_{\mu}$. Let $f^{*}$ denote the function $x \rightarrow\left(f\left(x^{-1}\right)\right)^{-}$(the bar denotes complex conjugate) and $\mu^{*}$ the measure $f \rightarrow\left(\mu\left(f^{*}\right)\right)^{-}$. Let furthermore $J$ denote the involution $f \rightarrow f^{*}$. Then it follows by routine computations that

$$
L_{\mu^{*}}=J R_{\mu} J \text {. }
$$

$L_{\mu^{*}}$ acting on $L^{q}(G)\left(p^{-1}+q^{-1}=1\right)$ is the adjoint of $L_{\mu}$ acting on $L^{p}(G)$, and similarly interpreted $R_{\mu}^{*}$ is the adjoint of $R_{\mu}$. If $p=q=2$ it follows easily that $L_{\mu}$ and $R_{\mu}$ have the same norm.

Operator norm will in general be denoted by \|\| and the adjoint of an operator $T$ by $T^{*}$. The domain of the operator will always be clear from the context since only bounded operators will be considered.

Definition 2.1. Let $f \in L^{1}(G)$ and let $L_{f}$ and $R_{f}$ be the operators on $L^{2}(G)$ defined above. The common value of $\left\|L_{f}\right\|$ and $\left\|R_{f}\right\|$ we shall call the spectral norm of $f$ and denote $\|f\|_{s p}$.

It is well known and easily proved that $\|f * g\|_{2} \leqq\|f\|_{1}\|g\|_{2}$ for all $g \in L^{2}(G)$ so that $\|f\|_{s p} \leqq\|f\|_{1}$, and consequently the spectral norm topology is weaker than the usual topology on $L^{1}(G)$. If $G$ is abelian we denote by $F$ the Fourier transform of $f, F(\chi)=\int f(x)(\chi(x))^{-} d x$. For a suitably normalized Haar meas-

(2) See Weil [16, Ch. III ]. 
ure $d \chi$ on the dual group $\hat{G}$ the Plancherel formula $\int|f(x)|^{2} d x=\int|F(\chi)|^{2} d \chi$ is valid. As a simple consequence we derive the relation $\|f\|_{s p}=\max _{\chi}|F(\chi)|$ for all $f \in L^{1}(G)$.

Definition 2.2. A linear transformation $T$ on $L^{1}(G)$ is called spectrally continuous if it is continuous from the spectral norm topology to the $L^{1}$-topology. Equivalently $T$ is spectrally continuous if and only if $\sup _{\|f\| s p \leq 1}\|T f\|_{1}$ $<\infty$. Such a transformation is a bounded operator on $L^{1}(G)$ in the usual sense due to the inequality $\|f\|_{s p} \leqq\|f\|_{1}$.

Throughout this paper $\hat{G}$ shall denote the set of all equivalence classes of irreducible unitary representations of $G$. If $\chi$ is a member of $\hat{G}$ we denote by $U_{x}$ a member of the class $\chi$ acting on the $d_{x}$ dimensional Hilbert space $\mathfrak{K}_{\chi}$. If $G$ is compact and for some basis in $\mathfrak{F}_{x}, U_{x}$ has the matrix form $U_{x}=\left(u_{i j}^{x}\right)$, the Schur orthogonality relations together with the Peter-Weyl theorem express that the system of functions $\left(d_{x}\right)^{1 / 2} \cdot u_{i, j}^{\chi}, \chi \in \hat{G}, i, j=1,2, \cdots, d_{x}$ is a complete orthonormal system in $L^{2}(G)$. For each integer $n>0, U(n)$ denotes the compact group of all unitary operators on an $n$-dimensional Hilbert space.

For every locally compact space $S$ we let $\mathfrak{C}^{\infty}(S)$ denote the set of all complex valued bounded continuous functions on $S$ and $\mathcal{K}(S)$ the subset of $\mathfrak{e}^{\infty}(S)$ consisting of all functions vanishing outside some compact subset of $S$. The space $\mathfrak{e}(S)$ of continuous functions vanishing at infinity on $S$ is the uniform closure of $\mathfrak{K}(S)$ in $\mathcal{C}^{\infty}(S)$.

3. The theorems. We wish to determine all spectrally continuous operators on $L^{1}(G)$ that commute with the right translations $R_{a}, a \in G$. The answer is given by the two following theorems.

Theorem A. Let $G$ be a compact group. The spectrally continuous operators $T$ on $L^{1}(G)$ commuting with right translations on $G$ are precisely the left convolution operators $T=L_{f}$ with $f \in L^{2}(G)$.

Furthermore $(1 / 2)^{1 / 2}\|f\|_{2} \leqq\|T\| \leqq\|f\|_{2}$.

Let us look more closely at the case when $G$ is the circle group. If $f \in L^{1}(G)$ and $f(x) \sim \sum a_{n} e^{i n x}$ is the usual Fourier series representation of $f$, then as noted earlier we have $\|f\|_{s p}=\max _{n}\left|a_{n}\right|$. Suppose $\sum a_{n} e^{i n x}$ has the property that for each sequence $\gamma=\left(\gamma_{n}\right)$ tending to 0 (that is $\gamma=\left(\gamma_{n}\right) \in \mathrm{C}(Z)$ ) the series $\sum a_{n} \gamma_{n} e^{i n x}$ is a Fourier series for a function $f_{\gamma} \in L^{1}(G)$. It is trivial to verify that the mapping $T: \gamma \rightarrow f_{\gamma}$ of $\mathfrak{e}(Z)$ in to $L^{1}(G)$ is linear and has a closed graph $\left\{\left(\gamma, f_{\gamma}\right) \mid \gamma \in \mathfrak{e}(Z)\right\}$ in the product space $\mathfrak{e}(Z) \times L^{1}(G)$. Using the closed graph theorem of Banach we conclude that $T$ is continuous. Hence $T$ is a spectrally continuous operator on $L^{1}(G)$ which clearly commutes with translations on $G$ so Theorem A tells us that $\sum\left|a_{n}\right|^{2}<\infty$. This sharpens the theorem of Littlewood stated in the introduction, because each $\gamma \in \mathcal{C}(Z)$ can be written $\gamma=\alpha+\beta$ where $\alpha$ and $\beta$ are two sequences $\left(\alpha_{n}\right)$ and $\left(\beta_{n}\right)$ for which $\left|\alpha_{n}\right|$ and $\left|\beta_{n}\right|$ are independent of $n$. 
THEOREM B. Let $G$ be noncompact connected separable unimodular locally compact group. Then every spectrally continuous operator $T$ on $L^{1}(G)$ that commutes with the right translations on $G$ is 0 .

REmark. If $G$ is abelian one of the conditions "connected," "separable" in Theorem B is unnecessary. Both of them can be dropped if $\hat{G}$ is assumed to be connected. These statements are proved in [6].

In the proofs of Theorems A and B we make use of the following simple lemma which is well known to workers in the field $\left({ }^{3}\right)$.

LeмmA 3.1. The bounded operators on $L^{1}(G)$ that commute with the right translations on $G$ are precisely the operators $L_{\mu}$ where $\mu$ is a bounded measure.

Proof. Let $T$ be an operator on $G$ with the stated property. Since $T R_{a}$ $=R_{a} T, a \in G$ we see from $\S 2$ that $R_{a^{-1}} T^{*}=T^{*} R_{a^{-1}}$ for all $a \in G . T^{*}$ is a bounded operator on $L^{\infty}(G)$ and from the fact that $T^{*}$ commutes with right translations it follows easily that if $f$ is continuous and has compact support then $T^{*} f$ is continuous (even uniformly continuous). The functional $f \rightarrow\left(T^{*} f\right)(e)$ is clearly continuous in the uniform topology on $\mathscr{K}(G)$. Hence there exists a bounded measure $\nu$ on $G$ such that

$$
\left(T^{*} f\right)(e)=\int_{G} f(x) d \nu(x) .
$$

Since $T^{*}$ commutes with right translations on $G$ it follows that $T^{*}=L_{\nu^{\prime}}$ where $\nu^{\prime}(E)=\nu\left(E^{-1}\right)$ for each Borel set $E$. Hence $T=L_{\mu}$ with $\mu=\left(\nu^{\prime}\right)^{*}$. On the other hand it was mentioned in $\$ 2$ that every $L_{\mu}$ has the properties stated in the lemma.

4. The compact case. Proof of Theorem A.

We shall use the Peter-Weyl theory on $G$. Let $f$ be an arbitrary function in $L^{1}(G)$. For each irreducible unitary representation $U_{x}$ of $G$ on the $d_{x^{-}}$ dimensional Hilbert space $\mathcal{H}_{x}$ we define the endomorphism $A_{x}$ by $A_{x}$ $=\int f(x) U_{x}\left(x^{-1}\right) d x$ (the Fourier coefficient corresponding to $U_{x}$ ) and to $f$ we associate the Fourier series

$$
f(x) \sim \sum_{\chi \in \widehat{T}_{\tilde{\tau}}} d_{\chi} \operatorname{Tr}\left\langle A_{\chi} U_{\chi}(x)\right\rangle \quad(\operatorname{Tr}=\text { Trace })
$$

$f$ is uniquely determined by this Fourier expansion. The Peter-Weyl theorem says that

$$
\int_{G}|f(x)|^{2} d x=\sum_{\chi \in \widehat{G}} d_{\chi} \operatorname{Tr}\left(A_{\chi} A_{\chi}^{*}\right)
$$

${ }^{3}$ Added in proof. After this was written I have in fact found a proof of this lemma in H. W. Wendel's paper, Left centralizers and isomorphisms of group algebras, Pacific J. Math. vol. 2 (1952) pp. 251-261. The proof above is somewhat simpler. 
Here the finiteness of one side implies finiteness of the other.

If $g \in L^{2}(G)$ it is trivial to verify the inequality $\|f * g\|_{2} \leqq \max _{\mathbf{x}}\left\|A_{\mathbf{x}}\right\| \cdot\|g\|_{2}$ which shows that $\|f\|_{s p} \leqq \max _{\chi}\left\|A_{\chi}\right\|$. We have written here max instead of sup which is legitimate, since the function $\left\|A_{x}\right\|$ vanishes at infinity on the discrete space $\hat{G}$. Furthermore we have the relation

$$
\|f\|_{s p}=\max _{\boldsymbol{x} \in \widehat{G}}\left\|A_{\boldsymbol{\chi}}\right\| .
$$

Indeed, let $\left\|A_{\chi}\right\|$ reach its maximum for $\chi=\phi$. Then we have clearly for each endomorphism $B_{\phi}$ on $\mathfrak{K}_{\phi}$

$$
\left(\|f\|_{s p}\right)^{2} \geqq \operatorname{Tr}\left\{A_{\phi} A_{\phi}^{*} B_{\phi} B_{\phi}^{*}\right\}\left[\operatorname{Tr}\left(B_{\phi} B_{\phi}^{*}\right)\right]^{-1} .
$$

We choose a basis in $\mathfrak{K}_{\phi}$ for which $A_{\phi} A_{\phi}^{*}$ is represented by a diagonal matrix and then choose $B_{\phi}$ with matrix representation having all entities 0 except at the place in the diagonal where the largest eigenvalue of $A_{\phi} A_{\phi}^{*}$ occurs. The relation (4.1) follows immediately.

If $f \in L^{2}(G)$ and $g$ has Fourier coefficients $B_{\chi}$ we have evidently

$$
\begin{aligned}
\|f * g\|_{1} & \leqq\|f * g\|_{2} \\
& \leqq \max _{x}\left\|B_{x}\right\|\|f\|_{2} .
\end{aligned}
$$

Hence $L_{f}$ is spectrally continuous and the right inequality in Theorem A follows.

To prove the converse (and the nontrivial) part of Theorem A we assume that $T$ is a spectrally continuous operator on $L^{1}(G)$ commuting with the right translations $R_{a}, a \in G$. By Lemma 3.1 we have

$$
T g=\mu * g \quad \text { for all } g \in L^{1}(G),
$$

where $\mu$ is a bounded measure. To $\mu$ we assign the Fourier-Stieltjes series

$$
\mu(x) \sim \sum_{x \in \widehat{G}} d_{\chi} \operatorname{Tr}\left\langle A_{\chi} U_{\chi}(x)\right\rangle .
$$

This formalism merely expresses that for each $\chi \in \hat{G}$,

$$
A_{x}=\int U_{x}\left(x^{-1}\right) d \mu(x),
$$

where $U_{\chi}$ is an irreducible unitary representation from the class $\chi$. We remark that the series above in general has more than countably many nonvanishing terms. Furthermore we note that the measure $\mu$ is uniquely determined by its Fourier-Stieltjes series, because if all $A_{x}=0$ then for each $f \in L^{1}(G), f * \mu$ has a vanishing Fourier series, hence $f * \mu=0$ for all $f \in L^{1}(G)$ and $\mu=0$.

Now let $\chi_{1}, \chi_{2}, \cdots, \chi_{N}$ be an arbitrary finite subset of $\hat{G}$ and $U_{1}, U_{2}, \cdots$ $U_{N}$ corresponding representations of $G$. Then, using (4.1) we get 


$$
\begin{aligned}
\|T\| & =\sup _{\|\cdot\|_{s} p \leqq 1}\|\mu * g\|_{1} \geqq \sup _{V_{n} \in U\left(d_{n}\right)} \int_{G}\left|\sum_{1}^{N} d_{n} \operatorname{Tr}\left\langle A_{n} V_{n} U_{n}(x)\right\rangle\right| d x \\
& \geqq \int_{U\left(d_{1}\right) \times \cdots \times U\left(d_{N}\right)} d V \int_{G}\left|\sum_{1}^{N} d_{n} \operatorname{Tr}\left\langle A_{n} V_{n} U_{n}(x)\right\rangle\right| d x \\
& =\int_{G} d x \int_{U\left(d_{1}\right) \times \cdots \times U\left(d_{N}\right)}\left|\sum_{1}^{N} d_{n} \operatorname{Tr}\left\langle A_{n} V_{n} U_{n}(x)\right\rangle\right| d V .
\end{aligned}
$$

Here $d V$ stands for the product measure $d V_{1} d V_{2}, \cdots, d V_{N}, d V_{n}$ being the normalized Haar measure on $U\left(d_{n}\right)$. Now, for all $x \in G, U_{n}(x)$ belongs to $U\left(d_{n}\right)$ and due to the invariance of $d V$ we have

$$
\begin{aligned}
\int_{G} d x \int_{U\left(d_{1}\right) \times \cdots \times U\left(d_{N}\right)} \mid \sum_{1}^{N} d_{n} & \operatorname{Tr}\left\langle A_{n} V_{n} U_{n}(x)\right\rangle \mid d V \\
& =\int_{U\left(d_{1}\right) \times \cdots \times U\left(d_{N}\right)}\left|\sum_{1}^{N} d_{n} \operatorname{Tr}\left(A_{n} V_{n}\right)\right| d V .
\end{aligned}
$$

We put $F\left(V_{1}, V_{2}, \cdots, V_{N}\right)=\sum_{1}^{N} d_{n} \operatorname{Tr}\left(A_{n} V_{n}\right)$ and intend to evaluate the integrals $\int|F|^{2} d V$ and $\int|F|^{4} d V$. The function $F$ is a continuous function on $U\left(d_{1}\right) \times \cdots \times U\left(d_{N}\right)$ and is easily seen to have the Fourier series

$$
F\left(V_{1}, \cdots, V_{N}\right) \sim \sum_{1}^{N} d_{n} \operatorname{Tr}\left(A_{n} V_{n}\right)
$$

and by the Schur-Peter-Weyl theorem

$$
\int_{U\left(d_{1}\right) \times \cdots \times U_{\left(d_{N}\right)}}\left|\sum_{1}^{N} d_{n} \operatorname{Tr}\left(A_{n} V_{n}\right)\right|^{2} d V=\sum_{1}^{N} d_{n} \operatorname{Tr}\left(A_{n} A_{n}^{*}\right)
$$

For the other integral we need the following

LeMma 4.1. Let $A$ be an arbitrary $n \times n$ complex matrix $(n \geqq 2)$. Then

$$
\int_{U(n)}|\operatorname{Tr}(A V)|^{4} d V=\frac{2}{n^{2}-1}\left\{\left[\operatorname{Tr}\left(A A^{*}\right)\right]^{2}-\frac{1}{n} \operatorname{Tr}\left(A A^{*} A A^{*}\right)\right\} .
$$

Proof. We begin by writing $A=P O_{1}$ where $P$ is positive definite and $O_{1}$ is unitary and then $B=O_{2} P_{2}^{-1}$ where $B$ is a diagonal matrix, $O_{2}$ is unitary. Clearly $\operatorname{Tr}\left(A A^{*}\right)=\operatorname{Tr}\left(B B^{*}\right)$ and since $d V$ is left and right invariant it suffices to prove the lemma when $A$ is a diagonal matrix. Assuming this being the case and letting $e_{1}, e_{2}, \cdots, e_{n}$ be the corresponding basis for the Hilbert space on which $A$ and $V$ act, we have

$$
\int_{U(n)}|\operatorname{Tr}(A V)|^{4} d V=\sum_{i, j, k, l} a_{i i} \bar{a}_{j i} a_{k k} \bar{a}_{l l} \int_{U(n)} v_{i i} \bar{v}_{j i} v_{k k} \bar{v}_{l l} d V
$$


where $a_{i i}=\left(A \boldsymbol{e}_{i}, \boldsymbol{e}_{i}\right)$ and $v_{i i}=\left(V \boldsymbol{e}_{i}, \boldsymbol{e}_{i}\right)$ (inner product). The terms in the sum above fall into three classes, namely, I: All indices $i, j, k, l$, are the same, II: The indices $i, j, k, l$, form two pairs and III: Among the indices $i, j, k, l$, at least one is different from the others.

The terms of the type III give no contribution to the integral $\int|\operatorname{Tr}(A V)|^{4} d V$; in fact let us for example consider the term $a_{11} a_{22} \bar{a}_{22} \bar{a}_{44} v_{11} v_{22} \bar{v}_{22} \bar{v}_{44}$. We denote by $U_{1}(n-1)$ the subgroup $\left\{T \in U(n) \mid T \mathbf{e}_{1}=e_{1}\right\}$ and by $U_{1,2}(n-2)$ the subgroup $\left\{S \in U_{1}(n-1) \mid S \mathbf{e}_{2}=\mathbf{e}_{2}\right\}$. Furthermore let $\sum_{n}$ denote the unit sphere in a complex $n$-dimensional Hilbert space; then the space $U(n) / U_{1}(n-1)$ of left cosets $\tilde{V}=V U_{1}(n-1)$ can be identified with $\sum_{n}$ and the space $U_{1}(n-1) / U_{1,2}(n-2)$ of left cosets $\tilde{T}=T U_{1,2}(n-2)$ can be identified with $\sum_{n-1}$. From the integration theory on coset spaces we have (denoting by $d \tilde{V}$ and $d \tilde{T}$ the unique normalized measures on $\sum_{n}$ and $\sum_{n-1}$ invariant under the action of $U(n)$ and $U_{1}(n-1)$ respectively)

$$
\begin{aligned}
& \int_{U(n)} v_{11} v_{22} \bar{v}_{22} \bar{v}_{44} d V=\int_{U(n)}\left(V \mathbf{e}_{2}, \mathbf{e}_{1}\right)\left|\left(V \mathbf{e}_{2}, \mathbf{e}_{2}\right)\right|^{2}\left(\mathbf{e}_{4}, V \mathbf{e}_{4}\right) d V \\
& =\int_{\Sigma_{n}} d \tilde{V} \int_{U_{1}(n-1)}\left(V T \boldsymbol{e}_{1}, \boldsymbol{e}_{1}\right)\left|\left(V T \mathbf{e}_{2}, \boldsymbol{e}_{2}\right)\right|^{2}\left(\boldsymbol{e}_{4}, V T \mathbf{e}_{4}\right) d T \\
& =\int_{\Sigma_{n}} d \tilde{V} \int_{\Sigma_{n-1}} d \tilde{T} \int_{U_{1,2}(n-2)}\left(V T S \boldsymbol{e}_{1}, \boldsymbol{e}_{1}\right)\left|\left(V T S \boldsymbol{e}_{2}, \boldsymbol{e}_{2}\right)\right|^{2}\left(\boldsymbol{e}_{4}, V T S \boldsymbol{e}_{4}\right) d S
\end{aligned}
$$

which vanishes since

$$
\int_{U_{1,2}(n-2)}\left\langle(V T)^{-1} \boldsymbol{e}_{4}, S \mathbf{e}_{4}\right\rangle d S=0
$$

due to the orthogonality relations for the canonical representation $J: S \rightarrow S$ of $U_{1,2}(n-2)$.

Since terms of type III give no contribution, we obtain

$$
\begin{aligned}
\int_{U(n)}|\operatorname{Tr}(A V)|^{4} d V= & \sum_{1}^{n}\left|a_{i i}\right|^{4} \int_{U(n)}\left|v_{i i}\right|^{4} d V \\
& +2 \sum_{i \neq j}\left|a_{i i}\right|^{2}\left|a_{j j}\right|^{2} \int_{U(n)}\left|v_{i i}\right|^{2}\left|v_{j i}\right|^{2} d V \\
& +\sum_{i \neq j} a_{i i}^{2} \bar{a}_{i j}^{2} \int_{U(n)} v_{i i} \bar{v}_{j i}^{2} d V,
\end{aligned}
$$

and it suffices to compute the integrals

$$
\int_{U(n)}\left|v_{11}\right|^{4} d V, \quad \int_{U_{(n)}}\left|v_{11}\right|^{2}\left|v_{22}\right|^{2} d V \text { and } \int_{U_{(n)}} v_{11}^{2} \bar{v}_{22}^{2} d V \text {. }
$$


Using the previous notation we have

$$
\begin{aligned}
\int_{U(n)}\left|v_{11}\right|^{4} d V & =\int_{U(n)}\left|\left(V \mathbf{e}_{1}, \mathbf{e}_{1}\right)\right|^{4} d V=\int_{\Sigma_{n}} d \tilde{V} \int_{U_{1}(n-1)}\left|\left(V T \mathbf{e}_{1}, \mathbf{e}_{1}\right)\right|^{4} d T \\
& =\int_{\Sigma_{n}}\left|\left(V \mathbf{e}_{1}, \mathbf{e}_{1}\right)\right|^{4} d \tilde{V}=\int_{\left|z_{1}\right|^{2}+\cdots+\left|z_{n}\right|^{2}=1}\left|z_{1}\right|^{4} d \tilde{V} .
\end{aligned}
$$

Let now $j_{1}, k_{1}, \cdots, j_{n}, k_{n}$ be a basis for a $2 n$-dimensional euclidean vector space. The mapping

$$
\sum_{1}^{n} \alpha_{p} e_{p} \rightarrow \sum_{1}^{n} \frac{1}{2}\left(\alpha_{p}+\bar{\alpha}_{p}\right) j_{p}-\frac{1}{2} i\left(\alpha_{p}-\bar{\alpha}_{p}\right) k_{p}
$$

is an isometric mapping of the $n$-dimensional Hilbert space onto the $2 n$ dimensional real euclidean space. $\sum_{n}$ is mapped onto the real $(2 n-1)$ dimensional sphere $S^{2 n-1}$ and the measure $d \tilde{V}$ on $\sum_{n}$ is transformed into the unique normalized measure $d \omega$ on $S^{2 n-1}$ which is invariant under the orthogonal group $\boldsymbol{O}(2 n)$.

Now we clearly have

$$
\int_{\Sigma_{n}}\left|z_{1}\right|^{4} d V=\int_{x_{1}+\cdots+x_{2 n}=1}^{2}\left(x_{1}^{2}+x_{2}^{2}\right)^{2} d \omega=2 \int_{S^{2 n-1}} x_{1}^{4} d \omega+2 \int_{S^{2 n-1}} x_{1}^{2} x_{2}^{2} d \omega
$$

and if $\Omega\left(S^{m}\right)$ denotes the euclidean surface area of the real $m$-dimensional unit sphere we get

$$
\int_{S^{2 n-1}} x_{1}^{4} d \omega=\frac{1}{\Omega\left(S^{2 n-1}\right)} \int_{-1}^{1} x_{1}^{4} \Omega\left(S^{2 n-2}\right)\left(1-x_{1}^{2}\right)^{n-1}\left(1-x_{1}^{2}\right)^{-1 / 2} d x_{1}
$$

and using the formula

$$
\Omega\left(S^{m}\right)=\frac{2 \pi^{(m+1) / 2}}{\Gamma((m+1) / 2)}
$$

the integral reduces to

$$
\int_{S^{2 n-1}} x_{1}^{4} d \omega=\frac{2\left(\pi^{2 n-1}\right)^{1 / 2}}{\Gamma(n-1 / 2)} \cdot \frac{\Gamma(n)}{2\left(\pi^{2 n}\right)^{1 / 2}} \cdot \frac{\Gamma(5 / 2) \Gamma(n-1 / 2)}{\Gamma(n+2)}=\frac{3}{4 n(n+1)} .
$$

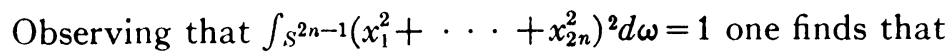

$$
\int_{S^{2 n-1}} x_{1}^{2} x_{2}^{2} d \omega=\frac{1}{4 n(n+1)}
$$

and therefore

$$
\int_{U(n)}\left|v_{11}\right|{ }^{4} d V=\frac{2}{n(n+1)}
$$


Using the notation introduced above we have

$$
\begin{aligned}
\int_{U(n)}\left|v_{11}\right|^{2}\left|v_{22}\right|^{2} d V & \left.=\int_{U_{(n)}} \mid V \mathbf{e}_{1}, \mathbf{e}_{1}\right)\left.\right|^{2}\left|\left(V \mathbf{e}_{2}, \mathbf{e}_{2}\right)\right|^{2} d V \\
& =\int_{\Sigma_{n}} d \tilde{V} \int_{U_{1}(n-1)}\left|\left(V T \mathbf{e}_{1}, \mathbf{e}_{1}\right)\right|^{2}\left|\left(V T \mathbf{e}_{2}, \mathbf{e}_{2}\right)\right|^{2} d T \\
& =\int_{\Sigma_{n}} d \tilde{V} \int_{\Sigma_{n-1}} d \tilde{T} \int_{U_{1,2}(n-1)}\left|\left(V T S \mathbf{e}_{1}, \mathbf{e}_{1}\right)\right|^{2}\left|\left(V T S \mathbf{e}_{2}, \mathbf{e}_{2}\right)\right|^{2} d S \\
& =\int_{\Sigma_{n}}\left|\left(V \mathbf{e}_{1}, \mathbf{e}_{1}\right)\right|^{2} d \tilde{V} \int_{\Sigma_{n-1}}\left|\left(T \mathbf{e}_{2}, V^{-1} \mathbf{e}_{2}\right)\right|^{2} d \tilde{T} .
\end{aligned}
$$

If we write

$$
\begin{aligned}
V \mathbf{e}_{1} & =w_{1} \mathbf{e}_{1}+\cdots+w_{n} \boldsymbol{e}_{n}, \\
V^{-1} \mathbf{e}_{2}= & b_{1} \boldsymbol{e}_{1}+\cdots+b_{n} \boldsymbol{e}_{n}, \\
T \mathbf{e}_{2}= & 0 \cdot \boldsymbol{e}_{1}+z_{2} \boldsymbol{e}_{2}+\cdots+z_{n} \boldsymbol{e}_{n} \\
& \quad\left(\text { since }\left(T \mathbf{e}_{2}, \boldsymbol{e}_{1}\right)=\left(\boldsymbol{e}_{2}, T^{-1} \boldsymbol{e}_{1}\right)=\left(\boldsymbol{e}_{2}, \boldsymbol{e}_{1}\right)=0\right.
\end{aligned}
$$

we obtain

$$
\begin{aligned}
\int_{U(n)}\left|v_{11}\right|^{2}\left|v_{22}\right|^{2} d V & =\int_{\left|w_{1}\right|^{2}+\cdots+\left|w_{n}\right|^{2}=1}\left|w_{1}\right|^{2} d \tilde{V} \\
& \cdot \int_{\left|z_{2}\right|^{2}+\cdots+\left|z_{n}\right|^{2}=1}\left|\bar{b}_{2} z_{2}+\cdots+b_{n} z_{n}\right|^{2} d \tilde{T} \\
= & (n-1)^{-1} \int_{\left|w_{1}\right|^{2}+\cdots+\left|w_{n}\right|^{2}=1}\left|w_{1}\right|^{2}\left(\left|b_{2}\right|^{2}+\cdots+\left|b_{n}\right|^{2}\right) d \tilde{V} \\
= & (n-1)^{-1} \int_{\Sigma_{n}}\left|w_{1}\right|^{2}\left(1-\left|w_{2}\right|^{2}\right) d \tilde{V}
\end{aligned}
$$

since $\left|b_{2}\right|^{2}+\cdots+\left|b_{n}\right|^{2}=1-\left|b_{1}\right|^{2}=1-\left|\left(V^{-1} \boldsymbol{e}_{2}, \mathbf{e}_{1}\right)\right|^{2}=1-\left|\left(\boldsymbol{e}_{2}, V \boldsymbol{e}_{1}\right)\right|^{2}=1$ $-\left|w_{2}\right|^{2}$. On the other hand

$$
\begin{aligned}
n(n-1) \int_{\Sigma_{n}}\left|w_{1}\right|^{2}\left|w_{2}\right|^{2} d \tilde{V} & =\int_{\Sigma_{n}}\left(\left|w_{1}\right|^{2}+\cdots+\left|w_{n}\right|^{2}\right)^{2} d \tilde{V}-n \int_{\Sigma_{\mathrm{n}}}\left|w_{1}\right|^{4} d \tilde{V} \\
& =1-n \frac{2}{n(n+1)}
\end{aligned}
$$

by (4.7) and (4.8). Hence

$$
\int_{U(n)}\left|v_{11}\right|^{2}\left|v_{22}\right|^{2} d V=\frac{1}{(n-1)(n+1)} .
$$


Finally we have by the same arguments

$$
\begin{aligned}
\int_{U(n)} v_{v_{11} \bar{v}_{22}^{2} d V}^{2} & =\int_{U_{(n)}}\left(V \mathbf{e}_{1}, \boldsymbol{e}_{1}\right)^{2}\left(\mathbf{e}_{2}, V \mathbf{e}_{2}\right)^{2} d V \\
& =\int_{\Sigma_{n}} w_{1}^{2} d \tilde{V} \int_{U_{1}(n-1)}\left(\boldsymbol{e}_{2}, V T \mathbf{e}_{2}\right)^{2} d T \\
& =\int_{\Sigma_{n}} w_{1}^{2} d \tilde{V} \int_{\Sigma_{n-1}}\left(b_{2} \bar{z}_{2}+\cdots+b_{n} \bar{z}_{n}\right)^{2} d \tilde{T} \\
& =0 .
\end{aligned}
$$

For later use we remark that the same methods yield the relations

$$
\int_{U(n)} v_{i i} v_{j j} d V=0 \quad \text { for } i, j \text {, arbitrary. }
$$

On substituting the found results in (4.6), Lemma 4.1 follows immediately.

The lemma has the following well known

COROLlary. Let $J$ denote the canonical representation $U \rightarrow U$ of $U(n)$. If $n \geqq 2$ the tensor product $J \otimes J$ decomposes into two irreducible components neither of which is the identity representation.

Proof. Denoting the character of a representation $T$ by $\chi_{T}$ we have $\chi_{J} \otimes_{J}=\chi_{J} \cdot \chi_{J}$ so the lemma gives the relation

$$
\int_{U(n)}\left|\chi_{J} \otimes J(V)\right|^{2} d V=2
$$

which implies the first statement of the corollary. Furthermore, the number of times the identity representation occurs in $J \otimes J$ is

$$
\int_{U(n)} \chi_{J} \otimes_{J}(V) d V=\int_{U_{(n)}}\left\{\chi_{J}(V)\right\}^{2} d V=0
$$

by $(4.10)$.

This decomposition of $J \otimes J$ corresponds to the decomposition of a covariant tensor of second order into a symmetric and a skew-symmetric part.

We can now estimate

$$
\int_{U\left(d_{1}\right) \times \cdots \times U\left(d_{N}\right)}\left|F\left(V_{1}, \cdots, V_{N}\right)\right|^{4} d V
$$

where $F\left(V_{1}, \cdots, V_{N}\right)=\sum_{1}^{N} d_{n} \operatorname{Tr}\left(A_{n} V_{n}\right)$. We expand the fourth power and note that terms of the type $\left|\operatorname{Tr}\left(A_{1} V_{1}\right)\right|^{2} \operatorname{Tr}\left(A_{2} V_{2}\right) \operatorname{Tr}\left(\bar{A}_{4} \bar{V}_{4}\right)$ give no contribution to the integral over $U\left(d_{1}\right) \times \cdots \times U\left(d_{N}\right)$ due to the orthogonality relations. Therefore 


$$
\begin{aligned}
\int_{U\left(d_{1}\right) \times \cdots \times U\left(d_{n}\right)} \mid & \left.\sum_{1}^{N} d_{n} \operatorname{Tr}\left(A_{n} V_{n}\right)\right|^{4} d V=\sum_{1}^{N} d_{n}^{4} \int_{U(n)}\left|\operatorname{Tr}\left(A_{n} V_{n}\right)\right|^{4} d V_{n} \\
& +2 \sum_{i \neq j} \int_{U\left(d_{i}\right)}\left|d_{i} \operatorname{Tr}\left(A_{i} V_{i}\right)\right|^{2} d V_{i} \int_{U\left(d_{j}\right)}\left|d_{i} \operatorname{Tr}\left(A_{j} V_{j}\right)\right|^{2} d V_{j} \\
& +\sum_{i \neq j} \int_{U\left(d_{i}\right)}\left\{d_{i} \operatorname{Tr}\left(A_{i} V_{i}\right)\right\}^{2} d V_{i} \int_{U\left(d_{j}\right)}\left\{d_{j} \operatorname{Tr}\left(\bar{A}_{j} \bar{V}_{j}\right)\right\}^{2} d V_{j} .
\end{aligned}
$$

The first sum on the right side we estimate by Lemma 4.1. The second sum can be directly evaluated by means of the orthogonality relations and the last sum vanishes due to the corollary. It is an elementary exercise to show that

$$
2 n^{2} /\left(n^{2}-1\right)\left\{\left[\operatorname{Tr}\left(A A^{*}\right)\right]^{2}-(1 / n) \operatorname{Tr}\left(A A^{*} A A^{*}\right)\right\} \leqq 2\left[\operatorname{Tr}\left(A A^{*}\right)\right]^{2}
$$

so we finally obtain

$$
\begin{aligned}
& \int_{U\left(d_{1}\right) \times \cdots \times U\left(d_{N}\right)}\left|\sum_{1}^{N} d_{n} \operatorname{Tr}\left(A_{n} V_{n}\right)\right|^{4} d V \\
& \leqq 2 \sum_{1}^{N} d_{n}^{2}\left[\operatorname{Tr}\left(A_{n} A_{n}^{*}\right)\right]^{2}+2 \sum_{i \neq j} d_{i} d_{j} \operatorname{Tr}\left(A_{i} A_{i}^{*}\right) \operatorname{Tr}\left(A_{i} A_{j}^{*}\right)
\end{aligned}
$$

and using (4.5)

$$
\int\left|F\left(V_{1}, \cdots, V_{N}\right)\right|^{4} d V \leqq 2\left[\int\left|F\left(V_{1}, \cdots, V_{N}\right)\right|^{2} d V\right]^{2} .
$$

By Hölders inequality we have

$$
\begin{aligned}
& {\left[\int\left|F\left(V_{1}, \cdots, V_{N}\right)\right| d V\right]^{2}} \\
& \qquad\left[\int\left|F\left(V_{1}, \cdots, V_{N}\right)\right|^{4} d V\right]^{-1}\left[\int\left|F\left(V_{1}, \cdots, V_{N}\right)\right|^{2} d V\right]^{3}
\end{aligned}
$$

and using (4.3), (4.4), (4.5) and (4.12) we finally obtain

$$
\frac{1}{2} \sum_{1}^{N} d_{n} \operatorname{Tr}\left(A_{n} A_{n}^{*}\right) \leqq\|T\|^{2} \text {. }
$$

The coefficients $A_{1}, \cdots, A_{N}$ were chosen arbitrarily from the FourierStieltjes series (4.2), and the relation (4.13) shows that this series is indeed a Fourier series for a function $f \in L^{2}(G)$. Since a measure is uniquely determined by its Fourier-Stieltjes series, the measure $\mu$ is absolutely continuous and has derivative $f$ with respect to Haar measure. Hence $T g=\mu * g=f * g$. Furthermore (4.13) shows that $(1 / 2)^{1 / 2}\|f\|_{2} \leqq\|T\|$ and Theorem $A$ is then completely proved. 
5. The noncompact case. Estimate of the spectral norm. We proceed to prove Theorem B, so let $T$ be a spectrally continuous operator on $L^{1}(G)$ which commutes with all the right translations $R_{a}, a \in G$. From Lemma 3.1 we know that $T$ is of the form

$$
T g=\mu * g
$$

and since $T$ is spectrany continuous there exists a constant $M>0$ such that

$$
\|\mu * g\|_{1} \leqq M\|g\|_{8 p} \quad \text { for all } g \in L^{1}(G) .
$$

We wish to show that $\mu=0$ and for this purpose we can assume $\mu$ absolutely continuous with respect to Haar measure. In fact (5.1) implies

$$
\|f * \mu * g\|_{1} \leqq M\|f\|_{1}\|g\|_{s p} \quad \text { for all } f, g \in L^{1}(G)
$$

and $f * \mu \in L^{1}(G)$. Furthermore, if $f * \mu=0$ for all $f \in L^{1}(G)$ then $\mu=0$.

Assuming now $\mu$ absolutely continuous, let $h$ be the derivative of $\mu$. To show that the relation

$$
\|h * g\|_{1} \leqq M\|g\|_{s p} \quad \text { for all } g \in L^{1}(G)
$$

implies $h=0$, we use the generalized Plancherel formula due to Segal and Mautner $[14 ; 15]$ and [8]. This formula can be described as follows.

Let $G$ be a separable unimodular group and put $\mathfrak{H C}=L^{2}(G)$. We consider the operators $L_{f}$ and $R_{f}$ on $\mathfrak{F}$ for all $f \in L^{1}(G)$. Let $\mathscr{L}$ and $R$ denote the weak closures (in the set of all bounded operators on $\mathfrak{H}$ ) of the sets $\left\{L_{f} \mid f \in L^{1}(G)\right\}$ and $\left\{R_{f} \mid f \in L^{1}(G)\right\}$ respectively. Then $\mathscr{L} \cap \mathcal{R}$ is the center of each algebra $\mathscr{L}$ and $\Omega$ (Segal [13], Godement [4]). If $\Gamma$ denotes the maximal ideal space of the commutative Banach algebra $\& \cap \Re$, there exists a measure $\mu$ on $\Gamma$ such that $\mathfrak{H}, \mathcal{L}$ and $\mathbb{R}$ can be written as a direct integral $\left({ }^{4}\right)$ with respect to the center $\mathscr{L} \cap R$ :

$$
\begin{gathered}
\mathcal{H}=\int_{\mathrm{\Gamma}} \mathfrak{F C}_{\gamma} d \mu(\gamma), \\
\mathscr{L}=\int_{\mathrm{I}} \mathfrak{L}_{\gamma} d \mu(\gamma), \quad \mathbb{R}=\int_{\mathrm{r}} \mathfrak{R}_{\bar{\gamma}} d \mu(\gamma)
\end{gathered}
$$

where for each $\gamma \in \Gamma, \mathfrak{L}_{\gamma}$ and $\mathfrak{R}_{\gamma}$ are rings of operators acting on the Hilbert space $\mathfrak{H}_{\gamma}$.

For almost all $\gamma \in \Gamma$ there is a two sided( $\left(^{5}\right)$ irreducible unitary representation $\left\{L_{\gamma}, R_{\gamma}\right\}$ on $\mathcal{F}$ such that for $f \in L^{1}(G)$ the decomposition of $L_{f}$ and $R_{f}$ according to (5.3) is given by

(4) For the definition and further details, see von Neumann [12], Segal [15] and Godement

(5) For this notion see Godement [3] and Segal [15]. 


$$
\begin{aligned}
& L_{f}=\int_{\Gamma} F_{\gamma} d \mu(\gamma) \text { where } F_{\gamma}=\int_{G} L_{\gamma}(x) f(x) d x ; \\
& R_{f}=\int_{\Gamma} F^{\gamma} d \mu(\gamma) \text { where } F^{\gamma}=\int_{G} R_{\gamma}(x) f(x) d x .
\end{aligned}
$$

For these (almost all) $\gamma, \mathfrak{L}_{\gamma}$ and $\mathbb{R}_{\gamma}$ are the weakly closed algebras generated by $L_{\gamma}(x), x \in G$ and $R_{\gamma}(x), x \in G$ respectively, and are therefore (due to the irreducibility of $\left.\left\{L_{\gamma}, R_{\gamma}\right\}\right)$ factors. We denote the relative dimension function in the factor $\mathscr{L}_{\gamma}$ by $\operatorname{dim}_{\gamma}$ and the corresponding relative trace by $\operatorname{Tr}_{\gamma}$. Furthermore we put $\operatorname{rank}_{\gamma}(A)=\operatorname{dim}_{\gamma}($ Range $A)$.

As usual we refer to the mapping $\gamma \rightarrow F_{\gamma}$ as the Fourier transform of $f$. The Plancherel formula asserts the existence of a strictly positive $\mu$-measurable function $a(\gamma)$ on $\Gamma$ such that

$$
\int_{i_{i}}|f(x)|^{2} d x=\int_{\Gamma} \operatorname{Tr}_{\gamma}\left(F_{\gamma} F_{\gamma}^{*}\right) a(\gamma) d \mu(\gamma) \quad \text { for each } f \in L^{1}(G) \cap L^{2}(G) .
$$

Lemma 5.1. If $f \in L^{1}(G) \cap L^{2}(G)$ and $F_{\gamma}$ is the Fourier transform of $f$, we have the inequality

$$
\|f\|_{s p} \leqq \underset{\gamma \in \Gamma}{\operatorname{ess} . \sup }\left\|F_{\gamma}\right\| .
$$

Proof. From the definition of the spectral norm it is clear that

$$
\|f\|_{s p}=\sup _{\sigma \in \mathcal{D},\|\ell\|_{2} \leqq 1}\|f * g\|_{2}
$$

where $\mathfrak{I}$ is an arbitrary dense subspace of $L^{2}(G)$. For $\mathfrak{D}$ we shall choose the set of all finite sums $\sum a_{i} f_{i}$ where $a_{i}$ are complex numbers, and $f_{i}$ are functions in $L^{2}(G)$ such that $f_{i}=f_{i}^{*}$ and $f_{i} * f_{i}=f_{i}$. As shown by Ambrose [1], D is indeed dense in $L^{2}(G)$. Now let $g=\sum a_{i} f_{i} \in \mathfrak{D}$ and denote by $G_{\gamma}$ and $F_{\gamma}^{i}$ the corresponding Fourier transforms. $F_{\gamma}^{i}$ is a projection almost everywhere so $\operatorname{Tr}_{\gamma}\left(F_{\gamma}^{i}\right)=\operatorname{dim}_{\gamma}\left(F_{\gamma}^{i}\right)$ and

$$
\int_{G}\left|f_{i}(x)\right|^{2} d x=\int_{\Gamma} \operatorname{dim}_{\gamma}\left(F_{\gamma}^{i}\right) a(\gamma) d \mu(\gamma) .
$$

Since $a(\gamma)>0, F_{\gamma}^{i}$ is for almost all $\gamma$ of finite $\gamma$-rank and the same holds for $G_{\gamma}$ and $G_{\gamma} G_{\gamma}^{*}$. Using simple formal properties of the relative trace (see von Neumann [11, Theorems I and II]) we obtain

$$
\begin{aligned}
\int_{G}|f * g(x)|^{2} d x & =\int_{\Gamma} \operatorname{Tr}_{\gamma}\left[F_{\gamma} G_{\gamma}\left(F_{\gamma} G_{\gamma}\right)^{*}\right] a(\gamma) d \mu(\gamma) \\
& \leqq \int_{\Gamma}\left\|F_{\gamma}^{*} F_{\gamma}\right\| \operatorname{Tr}\left(G_{\gamma} G_{\gamma}^{*}\right) a(\gamma) d \mu(\gamma) \leqq \text { ess. sup } \gamma\left\|F_{\gamma}\right\|^{2} \int_{G}|g(x)|^{2} d x
\end{aligned}
$$


which proves the lemma.

6. Completion of the proof. To prove Theorem $\mathrm{B}$ or that $h$ in (5.2) must be 0 we first assume that $h$ is continuous and has compact support. Making use of the connectedness of $G$ we then construct a sufficiently large class $\mathfrak{M}$ of approximate identities in $L^{1}(G)$ relative to $h$, that is functions $g \in L^{1}(G)$ for which $\|h * g\|_{1}$ is approximately equal to $\|h\|_{1}\left\|_{g}\right\|_{1}$ and such that $\sup _{o \in \mathfrak{N}}\|g\|_{1} /\|g\|_{s p}=\infty$. The inequality (5.2) will then show that $h=0$. Finally, the restriction that $h$ should belong to the class $\mathcal{K}(G)$ is removed by means of a suitable approximation argument.

Now assuming $h \in \mathscr{K}(G)$ and $h \neq 0$ we choose $\epsilon$ such that $0<\epsilon<\|h\|_{1}$ and a continuous function $u(x)$ vanishing outside a compact neighborhood of $e$ such that

$$
\|h * u-h\|_{1}<\epsilon \text { and }\|u\|_{1}=1 .
$$

Let $K$ be a compact subset of $G$ outside which all the functions $h, u$ and $h * u$ vanish. From a structure theorem of Iwasawa and Yamabe about connected locally compact groups (see e.g. Montgomery and Zippin [10, pp. 188-189]) it is easily deduced that there exists an element $d \in G$ such that the sets $K d^{n}, n=0, \pm 1, \pm 2, \cdots$ are all pairwise disjoint. Let $g$ be an arbitrary finite linear combination of the $d^{n}$-translates of $u$, i.e. $g(x)=\sum a_{n} u\left(x d^{n}\right)$. Then $h * g=\sum a_{n} R_{d^{n}}(h * u)$, and due to the construction of $d$ we have

$$
\|g\|_{1}=\sum\left|a_{n}\right| \text { and }\|h * g\|_{1}=\sum\left|a_{n}\right|\|h * u\|_{1}
$$

and in view of (6.1) it follows that $\|h * g\|_{1}=\|g\|_{1}\|h\|_{1}+\langle\epsilon\rangle\|g\|_{1}$ where $|\langle\epsilon\rangle|<\epsilon$. From (5.2) we conclude that

$$
\|g\|_{1} \leqq N\|g\|_{s p} \text { where } N \text { is a constant }
$$

for all $g$ of the form $g(x)=\sum a_{n} u\left(x d^{n}\right)$. Now let $G_{\gamma}$ be the Fourier transform of $g(x), G_{\gamma}=\int_{G} L_{\gamma}(x) g(x) d x$. We find immediately that $G_{\gamma}=U_{\gamma}\left[\sum a_{n} L_{\gamma}\left(d^{-n}\right)\right]$ where $U_{\gamma}$ is the Fourier transform of $u(x)$. Since $\left\|U_{\gamma}\right\| \leqq 1$ we get from Lemma 5.1 and $(6.3)$

$$
\sum\left|a_{n}\right| \leqq N \text { ess. sup }\left\|\sum a_{n}\left[L_{\gamma}(d)\right]^{-n}\right\| \leqq \sup _{U}\left\|\sum a_{n} U^{n}\right\|
$$

the supremum on the right being taken over all unitary operators on the spaces $\mathcal{K}_{\gamma}, \gamma \in \Gamma$. By the spectral theorem there exists a spectral measure $E$ on the real line such that $U=\int e^{i t} d E(t)$. Then it follows easily that

$$
\left\|\sum a_{n} U^{n}\right\| \leqq \sup _{t}\left|\sum a_{n} e^{i n t}\right|
$$

and from (6.4) we get

$$
\sum\left|a_{n}\right| \leqq N \sup _{i}\left|\sum a_{n} e^{i n t}\right|
$$

for arbitrary finitely many coefficients $a_{n}$. This inequality however, is impos- 
sible since there exist continuous functions with Fourier series that are not absolutely convergent. This contradiction shows that $h=0$.

We shall now free us of the restriction that $h$ has compact support. Let $h$ satisfy (5.2) and $h \in L^{1}(G),\|h\|_{1} \neq 0$. Let $\epsilon$ be a positive number less than $\|h\|_{1} / 4, k$ a continuous function on $G$ of compact support satisfying $\|h-k\|_{1}<\epsilon$. As before we can determine a function $u(x)$ and an element $d \in G$ such that all functions $g$ of the form $g(x)=\sum a_{n} u\left(x d^{n}\right)$ satisfy $\|k * g\|_{1}=\|k\|_{1}\|g\|_{1}$ $+\langle\epsilon\rangle\|g\|_{1}$. Hence

$$
\|k\|_{1}\|g\|_{1}-\epsilon\|g\|_{1} \leqq\|k * g\|_{1} \leqq\|(k-h) * g\|_{1}+\|h * g\|_{1} \leqq \epsilon\|g\|_{1}+M\|g\|_{8 p} .
$$

But this gives an inequality

$$
\|g\|_{1} \leqq R\|g\|_{s p} \quad \text { for all } g(x)=\sum a_{n} u\left(x d^{n}\right),
$$

and the same contradiction as before ensues.

\section{REFERENCES}

1. W. Ambrose, The $L_{2}$-system of a unimodular group. I, Trans. Amer. Math. Soc. vol. 65 (1949) pp. 26-48.

2. N. Bourbaki, Intégration, Chap. I-IV, Paris, 1952.

3. R. Godement, Mémoire sur la théorie des caractères dans les groupes localement compacts unimodulaires, J. Math. Pures Appl. vol. 30 (1951) pp. 1-110. 124.

4. - Sur la théorie des représentations unitaires, Ann. of Math. vol. 53 (1951) pp. $68-$

5. A. Grothendieck, Résultats nouveaux dans la théorie des opérations linéaires I-II, C.R. Acad. Sci. Paris vol. 239 (1954) pp. 577-579 and pp. 607-609.

6. S. Helgason, Multipliers of Banach algebras, Ann. of Math. vol. 64 (1956) pp. 240-254.

7. J. E. Littlewood, On the mean value of power series, Proc. London Math. Soc. vol. 25 (1924) pp. 328-337.

8. F. I. Mautner, Unitary representations of locally compact groups II, Ann. of Math. vol. 52 (1950) pp. 528-556.

10. D. Montgomery and L. Zippin, Topological transformation groups, New York, 1955.

11. J. von Neumann, On rings of operators III, Ann. of Math. vol. 41 (1940) pp. 94-161. 485 .

12. - On rings of operators. Reduction theory, Ann. of Math. vol. 50 (1949) pp. 401-

13. I. E. Segal, The two sided regular representation of a unimodular locally compact group, Ann. of Math. vol. 51 (1950) pp. 293-298.

14. - An extension of Plancherel's formula to separable unimodular groups, Ann. of Math. vol. 52 (1950) pp. 272-292.

15. Decompositions of operator algebras I, Memoirs of the American Mathematical Society vol. 9 (1951) pp. 1-67.

16. A. Weil, L'intégration dans les groupes topologiques et ses applications, Paris, 1950.

Massachusetts Institute of Technology,

Cambridge, Mass. 